\title{
La forge du droit. Naissance des identités juridiques en Europe $\left(I^{e}-X^{e} I I^{e}\right.$ siècles $)$
}

I. La construction de l'Europe suscite bien des difficultés notamment celle qu'il y a à définir son identité. Quels critères retenir ? Ni la géographie, depuis Hérodote, ni une prétendue langue originelle, ni la religion ne semblent pertinents, alors même que tradition, langue et religion furent, et sont encore en certaines parties du monde, considérées comme essentielles. Mais l'économie est la science maîtresse de notre époque et la Communauté européenne a longtemps reposé sur des objectifs et des valeurs essentiellement économiques. Avec les traités de Maastricht et d'Amsterdam est apparue la dimension politique et «sociétale » de la Communauté, devenue Union européenne. Le préambule du traité de l'UE comporte aujourd'hui un alinéa qui insiste sur l'héritage culturel, religieux et humaniste des pays membres. La référence aux « racines de l'Europe » est devenue courante, mais trop souvent elle sonne comme un passage obligé qui se réfère à une histoire convenue. Il n'est certes pas faux d'affirmer, comme le faisait par exemple Paul Valéry, que l'Europe s'est construite autour de trois influences : celles de la Grèce, de Rome et du christianisme. D'autres composantes ont cependant contribué à façonner l'Europe, comme l'a remarqué Karol Modzelewski, et toute une partie de son histoire est encore à faire, plus particulièrement son histoire juridique. C'est une partie de cette autre histoire, insistant sur les substrats coutumiers qui ont modelé, dans le long temps de l'histoire, le paysage normatif européen, qui fut l'objet d'un séminaire, organisé en 2013, conjointement par la Maison Française d'Oxford, placée alors sous la direction d'Anne Simonin, et All Souls college (University of Oxford), à l'initiative de Boudewijn Sirks (Regius Professor, All Souls college) et Soazick Kerneis (Paris Ouest - Maison Française d'Oxford) ; les actes de ce séminaire sont ici publiés grâce à la collaboration de Nathalie Kalnoky (chercheur au Centre d'Histoire et d'Anthropologie du droit, Université Paris Ouest). Les différentes contributions visaient à interroger, en l'historicisant, le legs du « droit romain », à remettre en cause le concept de coutume tel qu'il a été construit en droit romain, puis diffusé par la vulgate juridique, bref à remettre en cause $L e$ radici del mundo giuridico europeo, pour reprendre le titre de Maurizio Lupoi en 1994, traduit six ans plus tard en anglais aux Presses universitaires de Cambridge.

2. Sans doute, partout dans la «vieille Europe », l'influence du droit romain est sensible, diffusant une certaine représentation de la vie politique et juridique, centrée sur l'idée que l'individu, la personne, est, ou est supposé être, maître de ses actes. L'importance accordée au sujet de droit est manifeste dans la définition que donnait Ulpien de la justice, qui sera reprise dans les écoles de droit jusqu'au XIX ${ }^{\mathrm{e}}$ siècle : «La justice, c'est la ferme et constante volonté d'attribuer à chacun son droit ». Corrélativement à cet individualisme est posée la laïcisation du pouvoir : « Ce n'est pas la res publica qui est dans l'ecclesia, c'est l'ecclesia qui est dans la res publica », disait l'évêque Optat de Milev au IV ésiècle. À la faveur de la renaissance médiévale du droit romain, l'école de Bologne - la première université - mesura l'opportunité politique d'un droit capable d'affranchir le pouvoir de la théocratie. Telle fut la genèse de l' bomo juridicus et de l'appareil politique dans lequel il s'insère, habité et contraint par la norme, tel fut aussi le contexte romanisant dans lequel s'épanouirent les valeurs réaffirmées par les humanistes de la Renaissance, puis par les Lumières.

3. Tout cela est bien connu et nul ne songerait à le contester. On ne peut pour autant s'en tenir là et, depuis longtemps, au dogme d'une histoire totalement assujettie à une Antiquité triomphante, répondent des voix qui cherchent à faire entendre d'autres histoires. La liste est longue des chantres des Antiquités 
nationales qui, de Ronsard à Guillaume Postel, composent chacun à leur façon un roman des origines et l'on sait comment au XIX ${ }^{e}$ siècle, la controverse s'accusa entre germanistes et romanistes, trouvant une nouvelle dimension dans le contexte d'une Europe où les nations réinventaient leur passé.

4. « C'est la mémoire qui fait votre identité ; si vous avez perdu la mémoire, comment serez-vous le même homme $\gg$, disait Voltaire. Chacun connait l'importance de la mémoire dans la formation de l'homme, mais la mémoire, c'est aussi celle d'une communauté nationale comme l'ont bien montré Émile Durkheim et Maurice Halbwachs'. À une époque dévolue au culte de l'individu, où les processus de mondialisation fragilisent et les identités nationales et les gouvernements démocratiques, on pourrait croire que la mémoire fait place à l'oubli et que la marche vers un progrès capable de satisfaire les appétits individuels balaie les vestiges d'un passé devenu inutile, porteur de valeurs désuètes. Plutôt que de s'encombrer d'une mémoire collective, nombreux sont ceux que la vie contraint à tenter de recoller les fragments disloqués de leur mémoire individuelle. Mais la culture de la mémoire renvoie aussi au processus d'identification politique et il y a là un enjeu important qui n'a peut-être pas été suffisamment mesuré, une lacune qui pourrait expliquer les désillusions de notre siècle, ses difficultés à envisager son histoire et les dérives actuelles de ses utilisations. La mondialisation s'est accélérée, les frontières ont changé et cette modification des limites spatiales et géographiques n'est pas sans incidence sur les mentalités.

5. La question est importante, elle touche à celle de la construction de l'Europe. «Unis dans la diversité », aujourd'hui peut-être plus qu'hier la devise a un sens qui incite à reconsidérer la pluralité constitutive de l'Europe. La formation d'une identité européenne appelle-t-elle à l'épanouissement d'une mémoire européenne ? Les États membres, les nations, ou les groupes qui les composent ont chacun leur histoire ou leur pseudo-histoire, leur mémoire. Faut-il éliminer ces histoires, modéliser le passé, le formater pour parvenir à une mémoire homogène ? Ou laisser sombrer la mémoire dans les bruits du présent, en réduisant l'histoire à quelques commémorations spectaculaires et anachroniques ? Les difficultés des juristes à construire un droit européen illustrent la diversité des cultures juridiques, l'attachement des nations à leurs droits et aux traditions qui les fondent. La construction d'une «identité multiple » européenne passe par la promotion d'une culture commune, mais celle-ci peut-elle être commune sans prendre en considération les diverses traditions des peuples qui composent la communauté ? Sans l'étude de ces substrats, les valeurs affirmées risquent de ne relever que du discours et l'utilisation du passé satisfaire aux fantasmes. On sait combien le concept même d'identité appelle à précaution et quels usages erratiques peuvent en être faits. Mais on ne peut «jeter le bébé avec l'eau de son bain ». Si l'on veut promouvoir une culture juridique commune, il faut mesurer les spécificités nationales, les processus historiques complexes par lesquels se sont construits les droits de l'Europe, en bref, comme y invitent Frédéric Audren et Jean-Louis Halpérin, déconstruire les mythes qui emprisonnent les histoires juridiques nationales ${ }^{2}$.

6. La formation d'une véritable communauté en Europe suppose donc une meilleure connaissance de son patrimoine juridique complexe. Pour y parvenir, il faut envisager la façon dont fut conçue au XIX ${ }^{\mathrm{e}}$ siècle l'histoire des droits européens. Dans la perspective de l'histoire générale, se combinaient la recherche d'une perfection perdue et une conception évolutionniste centrée sur l'État et sur le droit conçu comme expression du pouvoir. Après la perte originelle, celle d'un modèle inégalable - il faut avoir lu ce que pense du droit romain un Ihering -, l'évolution allait, lentement, difficilement mais sûrement, vers un progrès qui était en fait un retour au passé. « L'homme marche vers son avenir mais à reculons ». Inévitablement, le passé modèle s'éloignait au fur et à mesure de la marche vers le progrès, reconstruit à chaque renaissance pour être un peu plus conforme, s'adaptant en fait au présent sans inquiétudes critiques. L'histoire était construite comme une diachronie inéluctable mais accidentée, une philogenèse où la naissance était au Proche-Orient ancien, l'âge d'or dans la Rome classique, tandis que le Moyen Âge, plus tard le seul Moyen Âge, passait pour une rupture, une parenthèse anarchique, la reconstruction d'un ordre dont nous étions

I M. Borgolte, «Memoria. Bilan intermédiaire d'un projet de recherche sur le Moyen Âge », dans Les tendances actuelles de l'histoire du Moyen Age en France et en Allemagne, J.-C. Schmitt \& O. G. Oexle éd., Paris, 2003, p. 64.

2 Fr. Audren \& J.-L. Halpérin, La culture juridique française. Entre mythes et réalités $X I X^{e}-X X^{e}$ siècles, Paris, 20I3, invitent à rompre avec la croyance en une tradition monolithique fondant une culture juridique uniforme et à considerer la pluralité des cultures juridiques des $\mathrm{XIX}^{\mathrm{e}}$ et $\mathrm{XX}^{\mathrm{e}}$ siècles en les replaçant dans le contexte de la diversité des foyers dont elles sont issues. 
les héritiers nécessaires. Le fil d'Ariane de cette analyse était le droit romain, outil du pouvoir, matrice et promoteur de l'unité juridique même si ce modèle était brouillé en Allemagne par la recherche des racines médiévales des « peuples » et en France par la prédominance de l'école de l'exégèse sur l'école historique. L'Italie songeait toujours à Rome. Le Royaume-Uni suivait sa voie propre, l'histoire du droit n'y existait pas en tant que telle, le droit était l'histoire, « la coutume immémoriale du peuple anglais ».

7. Pour éviter l'approche téléologique, mieux vaut prendre la question à rebours et insister sur la pluralité de l'héritage romain's Sur un même socle se sont développées des formes juridiques différentes, produits de processus d'acculturation variables. Rappelons d'abord comment la construction de la mémoire collective s'est bâtie sur des failles qui obèrent la réussite du programme européen (I). Pour comprendre les racines juridiques de l'Europe, il faut en passer par une micro-histoire, s'intéresser aux détails qui composent le vaste panorama des héritages de Rome (II).

\section{Les failles de la mémoire}

8. Aujourd'hui, la question des racines juridiques de l'Europe prend un nouveau sens du fait de l'affaiblissement de la culture latine et de l'influence grandissante des pays anglo-saxons dans le contexte politique d'une Union européenne centrée autour de l'axe Strasbourg-Bruxelles-Francfort mais qui regarde outre-Atlantique. L'explosion des recherches consacrées à l'Antiquité tardive et au Haut Moyen Âge, des périodes longtemps délaissées, est sans doute significative. Il faut trouver d'autres racines à l'Europe que le passé romain, nonobstant le fait que la géographie même de l'Empire, très orientalisante, soulève la délicate question de l'adhésion de la Turquie à l'Europe. Du point de vue des juristes, insister sur les racines germaniques de l'Europe présente l'avantage de relativiser l'apport du droit romain dans l'histoire du droit européen, un enjeu de taille dans la lutte d'influences qui oppose les systèmes continentaux (revendiquant l'héritage du droit romain) à ceux de Common Law, d'abord explicitement, puis aujourd'hui implicitement coutumiers. Quel peut être le poids de la loi - de l'héritage romain - face à la coutume et qui fait celle-ci, et pour qui ? La question est actuelle, comme l'illustrent les travaux poursuivis au sein de la Commission sur le droit européen des contrats, créée en 1980, sous l'égide du professeur danois Ole Landau. Sans aborder les contrats spécifiques, la commission Landau s'est efforcée de mener un travail d'harmonisation portant sur les principes contractuels. La question de l'harmonisation des droits et la recherche des principes fondamentaux s'imposent alors comme une des questions importantes à débattre.

9. Force est de constater que la construction d'un droit européen soulève beaucoup de réticences. Si l'enjeu économique rend concevable un droit commun de la vente, le projet d'un code civil européen passe pour menacer la souveraineté et l'identité des États. C'est qu'il se heurte à la diversité des traditions juridiques, à l'attachement sous-estimé des nations à leur droit. Il faut convenir que les droits nationaux sont des créations culturelles profondément enracinées. Lorsque les nations excipent de leur patrimoine culturel, il est frappant de constater la part qu'y tiennent les normes. Il y a là un phénomène récurrent que l'on peut illustrer à travers quelques exemples qui se rapportent à des périodes différentes. D’abord au VIe siècle, celui de Procope décrivant l'attachement d'anciens « soldats des Romains » - les Francs saliques - à leurs règles; ou bien celui du Kanun albanais, un code coutumier du $\mathrm{XV}^{e}$ siècle qui codifiait la vengeance, en usage jusqu'au régime communiste qui l'interdit, et dont la pratique contemporaine, en des formes abusives, accompagne un processus d'identification difficile; enfin, à l'heure ou le Royaume-Uni tend à se défaire, celui d'un timbre émis au Pays de Galles qui montre le « bon » roi Hywel Dda accompagné d'un juge, officiant un livre de droit - sa « loi » - à la main ; un érudit gallois du siècle écoulé, J. Goronwy

3 Sur la nécessité de déplacer l'angle de la recherche de « la force créatrice de tradition de la memoria » vers la perturbation introduite par les ruptures, Borgolte, ibid., p. 68, citant M. Foucault, Archéologie du savoir, Paris, 1969. Même constat de J.-L. Halpérin, ibid. p. 278-28o sur l'importance «d'une déconstruction de la vision "continuiste" du droit français à l'évolution linéaire depuis le Haut Moyen Âge et d'une réévaluation des moments de rupture ou de changement politicoculturel ». 
Edwards, l'avait déclaré : étudier les lois de Hywell Dda n'est pas seulement faire acte d'érudition, le droit, autant que la langue galloise, a constitué la nation. Ou encore la pierre du destin, où étaient jadis proclamés les rois écossais, prise par Edouard I en 1296 et insérée dans le trône du couronnement des rois d'Angleterre pour légitimer leur pouvoir sur le royaume conquis. Utilisée encore en 1953, elle fut en 1996 restituée solennellement à la garde de l'Écosse, le jour de la Saint-André, par la main du prince Andrew. On sait que l'union de l'Écosse au Royaume-Uni reste à l'ordre du jour. Bien d'autres exemples témoigneraient de la part prise par le droit et ses symboles dans la constitution des processus identitaires.

Io. Le processus de constitution des identités nationales est le produit d'une histoire complexe remontant à un passé très lointain et qui continue à hanter le présent. Prenons encore l'exemple de la GrandeBretagne. Comment expliquer que l'ancienne Britannia soit devenue en partie l'Angleterre d'aujourd'hui, alors même que les envahisseurs anglo-saxons étaient peu nombreux ? ... Ward-Perkins note les implications contemporaines de la représentation du passé telles qu'elles apparaissaient encore à la fin du XIX ${ }^{e}$ siècle, dans les manuels d'histoire destinés à l'éducation des enfants anglais :

The [British] women of course would be made slaves or they would sometimes be married to their masters. Thus there may doubtless be some little British and Roman blood in us, just as some few Welsh and Latin words crept into the English tongue from the very beginning. But we may be sure that we have not much of their blood in us, because we have so few of their words in our language... Now you will perhaps say that our forefathers were cruel and wicked men... And so doubtless it was... But... it has turned out much better in the end that our forefathers did thus kill or drive out nearly all the people whom they found in the land... [since otherwise] I cannot think that we should ever have been so great and free a people as we have been for many ages ${ }^{4}$.

\section{Reconstruire la mémoire}

II. À trop privilégier une histoire commune tenue et fabriquée par les milieux académiques, le risque est d'éloigner les peuples de leur histoire, de leur passé et de leur droit. Maîtriser le passé, c'est se donner les moyens de gérer le présent, de réconcilier les nations avec leur histoire, de leur redonner le goût du politique, en bref de refonder les bases du pacte social. Pour ce faire, il faut prêter attention aux microhistoires, donner de la profondeur au paysage politique des communautés nationales. Et il semble bien qu'un moment fondamental pour comprendre notre passé juridique soit celui qui se situe à la fin de l'Empire romain. C'est alors que se forment en Occident les premières nations (nationes) européennes, à partir des royaumes qui s'aménagent alors. La Res publica romana conquérante, après s'être imposée en Orient à des sociétés de «cités » de structure à peu près identique, avait entrepris d'intégrer les communautés tribales du Nord dans une formation politique de plus grande ampleur. Mais assez tôt, l'Empire dut renoncer au rêve d'une conquête illimitée. Le mouvement d'acculturation se continua pourtant. L'importation d'esclaves, achetés aux tribus extérieures, se maintint et l'Empire lui-même prit en main le gros de l'immigration en déportant de nombreuses communautés de diverses origines ethniques, hostiles ou réfugiées, pour les installer, avec une condition de dépendants publics (les dediticii) en Gaule septentrionale, dans le nord de l'Italie, dans les pays danubiens ou dans l'île de Bretagne. Finalement, des groupes armés plus compacts, aux ordres de condottieri barbares, furent acceptés bon gré mal gré et, dans la débâcle générale, certains de ces groupes prirent le pouvoir, administrant des regna. Ce processus aboutit, notamment en France, à une «mosaïque culturelle » qui se fixa en une carte anthropologique dont les couleurs contrastées vont très lentement se fondre dans l'unité administrative de l’Ancien Régime, elle-même oblitérée par les réformes du XIX ${ }^{e}$ siècle.

I2. Pour comprendre comment se sont formées les premières formes « nationales de droit », il faut donc remonter le courant de l'histoire. Alors que bien souvent il s'agissait, et il s'agit encore parfois, de montrer

4 E. A. Freeman, Old English History for Children, Londres, I869, 27-29, cité par B. Ward-Perkins, « Why Did the AngloSaxons Not Become More British ? », The English Historical Review, II5/462, 2000, p. 518. 
l'influence sur nos droits européens du droit romain stricto sensu - entendons par là le droit romain pensé et formulé par ses jurisconsultes -, il s'agira ici de s'arrêter sur les formes vulgaires du droit, qu'il s'agisse des pratiques populaires qui se sont développées dès l'Empire en marge du droit officiel, ou des avatars législatifs qui ont grandi après son effondrement, dans les premiers « gouvernements nationaux », les royaumes fort malencontreusement dits «barbares». Sans doute la reconstruction des droits qui a prévalu un peu partout au XIX ${ }^{e}$ siècle a sensiblement modifié le paysage juridique européen. Pour autant, le droit se construit par strates, et les conduites juridiques induites par les normes anciennes façonnent sans doute une part de «l'esprit des lois » présentes ou même futures. De même que la psychanalyse a montré l'importance d'un patrimoine psychique dans la construction du soi, l'histoire du droit doit révéler le patrimoine juridique des nations européenness. Dans un contexte politique où le régionalisme le dispute à la mondialisation, il est important que chaque communauté soit en paix avec son histoire, ses origines, comprenne les différentes étapes qui ont scandé son évolution. Construire ces mémoires permettrait d'éviter les dérives de l'histoire, les utilisations malhonnêtes du concept d'identité : montrer ainsi comment se sont enracinées les traditions juridiques, notamment la relation étroite qui a pu exister entre la loi et le privilège dans les sociétés anciennes, pourrait par exemple réconcilier les citoyens avec leur système juridique.

13. La reconstitution de ce substrat doit être menée à l'échelle européenne. Le poids de l'idéologie dans la construction des histoires nationales est bien connu. C'est en levant les frontières géographiques qui bornent les savoirs que l'on peut saisir le processus de fabrication des identités nationales, le comprendre et réfuter les utilisations idéologiques des pseudo-histoires. Pour saisir l'ampleur de l'héritage de Rome, il faut aussi renoncer à une grille de lecture où les données s'ordonneraient autour de couples antagonistes simplistes, romanité vs barbarie, ou romanisation vs résistance nationale ou bien encore loi vs coutumes. Plutôt que de raisonner en termes d'opposition, mieux vaut réaliser que c'est la richesse des processus d'acculturation que de susciter des formes nouvelles. Dans un contexte chaque fois différent, les traditions anciennes se mêlent pour produire des formes inédites qui parviennent à combiner l'apparemment inconciliable. C'est ainsi que les choses du passé, dans les habits neufs du présent, revivent pour longtemps.

I4. Un des traits saillants de cet héritage de la res publica romaine est la part prise par une littérature juridique administrative, des règlements qui concernaient la matière fiscale ou militaire consignés dans des livres à l'usage des administrateurs-juges en charge du contentieux. Du point de vue des juristes, sans doute ces livres n'étaient que de banals outils, des vade-mecum qui encadraient l'activité juridictionnelle des autorités administratives. Le style dépouillé en témoigne, qui tranche avec la rhétorique ampoulée de la vraie loi. Pour autant, dans la constitution de promulgation du code théodosien, deux exceptions sont prévues à l'exclusivité de la loi impériale, qui consacrent la validité de ces mesures, allant même jusqu'à leur donner valeur législative. Pour beaucoup, ces dispositions trouvaient leur fondement dans l'octroi de privilèges, coutumes fiscales ou lois particulières octroyées aux nations barbares de l'Empire. On mesure la valeur de la concession impériale qui donnait rang de loi à ces coutumes particulières. Elle incite à renouveler le sens même de ce qu'il faut entendre par loi au sortir de l'Empire, une loi qui a les pieds dans la pratique et qui bien souvent ressortit à l'ancienne catégorie des leges datae, des lois accordées par une autorité à des communautés.

I5. Au-delà de ces considérations qui concernent la méthode, la consécration de la valeur législative des privilèges administratifs fonde un rapport particulier des bénéficiaires à leurs lois. Prises dans leur acception fiscale, les coutumes déterminaient la solidarité du groupe, d'un point de vue technique l'obligation solidaire vis-à-vis de l'impôt, mais au-delà et de façon corrélative, le sentiment d'appartenance à une communauté qui ira croissant lorsque d'autres cadres publics disparaîtront. Lorsque la Rome impériale ne fut plus que souvenir, ce qui demeura c'est cette idée d'un régime particulier octroyé par le Prince. De la même façon, au fil du temps, les lois des nations ethniques devinrent vecteur d'identité. Ce ne fut pas d'abord la religion mais le droit qui donna aux débris de tribus installées dans l'Empire ce sentiment de commune appartenance qui leur permettait de se penser comme une même nation. Et parce

5 Voir notamment l'approche de P. Legendre, L'amour du censeur. Essai sur l'ordre dogmatique, Paris, 1974. 
que les barbares de l'Empire étaient d'abord des militaires, c'est dans l'armée que se forgèrent ces premières identités nationales, autour d'une même bannière, d'une même loi. La mission chrétienne vint ensuite, non sans mal.

I6. En fin de compte, les racines juridiques de l'Europe forment un entrelacs complexe. L'héritage du droit d'abord, composé du ius et de ses rhizomes, plus ou moins dépendants de la racine mère, enchevêtrement de règles qui réalisent l'adaptation des normes du droit romain aux réalités d'une nouvelle époque. Celui aussi des anciennes traditions accrochées aux discours mythiques des parentèles. Celles-ci ne se laissent guère saisir, tout juste lorsque la loi s'en empare pour les valider comme coutumes. Pour autant, même invisible, il faut rapporter cette pensée sauvage au processus de formation de notre droit. Parmi toutes les différences qui opposaient les traditions normatives héritées de Rome aux usages des sociétés coutumières, celle relative à l'usage des mots paraît fondamentale. Là où le ius accrochait le jugement à la règle et cultivait une certaine logique organisée autour de la sémantique juridique, la pensée coutumière se sert des mots pour voyager dans la nébuleuse du passé. Car les mots ne sont pas seulement les signifiants de la norme, chacun d'eux a son histoire qui elle-même en appelle bien d'autres, de sorte que les mots peuvent se déployer à l'infini. L'invocation à la norme procède alors d'un imaginaire au centre duquel les mots valent comme immémoriaux, porteurs de la pensée mythique. Dire la norme, c'est savoir voyager dans la forêt des mots, enfourcher celui qui mènera à la droite solution, une solution conforme à l'ordre ancien des choses. Au sortir de l'Empire, les voies du droit hésitaient donc entre un ius qu'encadraient des fictions, articulées autour des verba qu'il avait lui-même définis, et les sinuosités d'une pensée agrippée à des termes tutélaires, maillons de la vaste chaîne qui composait le grand Parler des anciens. Dans la Voie cheyenne, Karl N. Llewellyn et E. Alexandre Hoebel invitaient à goûter la saveur du droit. Qui veut pénétrer le premier Moyen Âge doit se convaincre que les fragrances de la loi y étaient multiples, ouvrir grandes « les portes de la perception » car la résolution des conflits sollicitait alors non pas tant le corps froid du droit, qu'un ensemble normatif qui puisait dans le registre des sens et se nourrissait de petits riens passés dans la mémoire du groupe.

I7. Finalement, que reste-t-il de ce lointain passé ? A-t-il définitivement disparu, mis à mal par les progrès de la raison? Les anthropologues l'ont bien montré, le long usage n'est qu'un mythe justificateur, le propre de l'usage étant d'évoluer. C'est précisément ce processus de transformation qu'il faut saisir si l'on veut comprendre comment le droit se crée. La tâche est complexe, parfois impossible mais il vaut la peine d'écouter les murmures du passé car ils ne sonnent pas le glas de cultures révolues. Dans le sillage de la coutume, s'esquissent déjà les formations du futur, celles des premières nations composant leurs solidarités à partir des reliefs de l'histoire.

17. Dans le même terreau travaillé par Rome se sont donc épanouies des formes juridiques différentes, produits de processus d'acculturation variables, des formes normatives qui ont survécu à l'Empire en Occident. D'une certaine façon, l'esprit de ce dossier n'est pas si éloigné, malgré une situation historique bien différente, de celui l'École historique allemande lorsque Savigny incitait à retrouver ce qui est propre à chaque nation, les traditions culturelles des peuples, ou qu'Henri Klimrath concevait son projet de géographie coutumière de la France qui devait saisir « les différences d'esprit juridique » des coutumes de la France. Mais là où le projet se focalisait sur les coutumes médiévales, nous croyons qu'il faut décaler l'enquête et la placer au moment de la dislocation de l'Empire romain, lors de l'établissement des premières « nations ». Le terme avait alors son sens originel « ceux qui sont nés ensemble » et désignait les sociétés dites barbares hors de l'Empire romain. Ces sociétés, ou du moins certaines d'entre elles, allaient être établies dans les cadres malmenés de la romanité impériale. Il s'agit ici d'observer le vaste processus d'acculturation juridique alors à l'œuvre et de comprendre la variété des formes qu'il revêtit. Les identités seront de la sorte comparables et le particulier pourra se réconcilier avec la communauté.

I8. On sait la place, depuis longtemps reconnue, de l'Église dans ce vaste mouvement. Les trois axes choisis pour tenter d'en esquisser une représentation un tant soit peu nouvelle tiennent compte des recherches les plus récentes. Ce sont d'abord l'armée qu'on a souvent tendance à séparer du droit par une application 
anachronique de l'adage cedant arma togae. Puis les restes non négligeables du droit impérial qui investit, ou tente d'investir les traditions vernaculaires. Et enfin, véhicules de cette influence romanisante, les écoles dont le rôle au Moyen Âge classique a depuis longtemps été mis en valeur, mais qui sont à l'œuvre depuis bien plus longtemps. Qu'on ne s'y trompe pas. Aucun de ces trois axes n'est véritablement séparé des autres. L'armée romaine relevait d'une administration militaire qui n'était pas sans lien avec les juristes du gouvernement central ; le droit romain qui influençait les Burgondes s'exerçait sous l'égide d'un magister militiae et l'influence du droit romain sur le Pays de Galles devait beaucoup à ce qui restait des écoles tenues par les clercs et ces écoles devaient composer avec des sociétés où l'activité guerrière avait bien souvent prévalu. Le découpage ici retenu ne vaut que comme grille de lecture.

\title{
I. L’armée et le droit
}

- Soazick Kerneis, « Rome and the Barbarians : the privilege of law / Rome et les barbares : le privilège du droit »

- Jean-Pierre Poly, "Freedom, warriors' bond, legal book. The Lex Salica between Barbarian custom and Roman law / Liberté, liens des guerriers, livre de droit. La lex salica entre coutume barbare et droit romain”

- Stefan Esders, « Late Roman Military Law in the Bavarian Code / Droit militaire romain tardif dans le code de Bavière »

\section{Entre deux mondes}

- Thomas Charles-Edwards \& Jaqueline Bemmer, «Irish and Welsh Law in the European Contexts / Droit irlandais et droit gallois dans le contexte européen »

- Ian Wood, «The Legislation of Magistri Militum: the laws of Gundobad and Sigismund / La législation des Magistri Militum : les lois de Gondebaud et Sigismond »

\section{L'école et le droit}

- Luca Loschiavo, «Isidore of Seville and the construction of a common legal culture in early medieval Europe / Isidore de Séville et la construction d'une culture juridique commune au début de l'Europe médiévale »

- Christophe Archan, «The five paths to a judge : an interpretation of Cóic Conara Fugill (Five Patbs to Judgement) / Les cinq chemins vers un juge : une interprétation du Cóic Conara Fugill (Cinq chemins du jugement) »

- Kees Bezemer, « Jacques de Revigny (d. 1296) : Roman law as a means to shape French law / Jacques de Revigny († 1296) : Le droit Romain comme moyen de façonner le droit français »

\author{
Soazick Kerneis \\ Université Paris Ouest - Maison française d'Oxford
}

